\title{
Investigation of the role of contact-induced vibrations in tactile discrimination of textures
}

\author{
Mariano Di Bartolomeo ${ }^{1,2,}$, Flavio Morelli ${ }^{1}$, Davide Tonazzi ${ }^{1,2}$, Francesco Massi $^{1,2}$, and Yves Berthier $^{2}$ \\ ${ }^{1}$ DIMA, Department of Mechanical and Aerospace Engineering, University of Rome La Sapienza, 00184 Rome, Italy \\ ${ }^{2}$ LaMCoS, Laboratoire de Mécanique des Contacts et des Structures, INSA de Lyon, 69621 Villeurbanne Cedex, France
}

Received: 20 April 2016 / Accepted: 13 June 2017

\begin{abstract}
Tactile perception of texture is meaningful for numerous industrial and social applications. While the sense of touch is provided by several contributing factors, each related to different sensory cutaneous units, textures with non-low spatial gradients can be appreciated only by the relative motion between the finger and the object. This work focuses on investigating the role that the vibrations induced during sliding contact play in the origin of tactile perception and surface texture discrimination. We shall focus in particular on the effect of the different spectral contents of the induced vibrations. To this end, first the vibration signals produced by touching different sample surfaces are recovered and analysed under controlled boundary conditions. Then, a collection of data from different subjects is recorded and reproduced by a dynamic exciter. Finally, the reproduced signals are randomly submitted to a panel of subjects in order to test their ability to distinguish the different surfaces only through their perception of the reproduced vibrations. The results, while highlighting the key role played by the spectral distribution of friction-induced vibrations, simultaneously reveal the limits of taking only vibrational signals into account if we are to achieve satisfactory recognition of the whole panel of tested textures.
\end{abstract}

Keywords: Tactile perception / friction induced vibration / texture / biomechanics / tribology

\section{Introduction}

Tactile perception, intended as the identification of the shape and texture of objects, is a result of an overall activity that involves primarily proprioceptors and mechanoreceptors [1]. Proprioceptors are receptors of kinesthesis, the perception of the position of the body, while mechanoreceptors are sensory cutaneous units. A better understanding of the mechanisms that contribute to tactile perception could provide useful recommendations for many industrial and social fields [2]: (a) development of interfaces for tele-operation systems, such as tele-diagnosis or microsurgery; (b) reproduction of touch perception (virtual reality); (c) increase of human perception (augmented reality); (d) development of tactile sensors for intelligent prostheses; (e) development of tests for evaluating tactile sensitivity during diagnosis or monitoring in rehabilitation processes; (f) design of tactile communication devices, smartphones and touchscreens; (g) the ergonomics of everyday objects, which significantly affects their commercial competitiveness and textile quality; and (h) identification of surface imperfections.

\footnotetext{
* e-mail: mariano.dibartolomeo@uniroma1.it
}

In the fields of acoustics and optics, the signals at the origin of stimuli are well known and commonly reproduced by acoustic speakers and monitors. However, the tactile sense is still largely unknown. Current literature addresses tactile perception in order both to understand the mechanical signals at the origin of stimuli and to reproduce those stimuli using tactile simulators. Recent works, for example, have focused either on the analysis of signals generated by touching everyday objects, like textile fabrics [3] or on their reproduction by simulators [4,5].

Surface tactile sensing involves different types of mechanoreceptive units, each consisting of an afferent fibre and its ending [6], located at a specific depth from the surface of the finger epidermis [7]. They recover the mechanical stimulus that comes from the contact between finger and touched surface, which is then transduced into an electric signal and sent to the brain. There are four main types of mechanoreceptors [8]: (a) Slowly adapting type I (SA I) afferents, which end in Merkel cells. These units react in a frequency range from 1 to $16 \mathrm{~Hz}$ and are particularly suited to the representation of surface shape and curvature [6]; (b) Fast adapting type I (FA I) afferents, which end in Meissner's corpuscles. The FA I units react from 3 to $60 \mathrm{~Hz}$. They are insensitive to static skin indentation but they respond well to dynamic skin deformation [9]; (c) Fast adapting type II (FA II) afferents, which end in Pacinian corpuscles. These 
respond within a broad frequency range $(40-500 \mathrm{~Hz})$ with a maximum sensitivity around $300 \mathrm{~Hz}$. They intensively filter low frequency stimuli and are particularly sensitive to transient stimulation [8,10]; and (d) Slowly adapting type II (SA II) afferents, which terminate in Ruffini endings. They are excited at a very low frequency range (a few $\mathrm{Hz}$ ). These units have a poor spatial resolution and play an important role in detecting skin stretch and in perceiving hand configuration and finger position [11].

In modern psychophysics and neurophysiology, the theory that tactile perception takes place by means of the so-called "duplex model" [12] is more and more accepted. This theory states that the tactile perception process occurs in different ways, depending on the presence of either fine (spatial period $<100 \mu \mathrm{m})$ or coarse $(>200 \mu \mathrm{m})$ textures [12-14]. The former are related mainly to friction induced vibrations (FIV), whereas the latter are more closely related to skin deformation gradients [13].

Taking the work of Katz as a starting point [12], modern studies have improved this theory by providing experimental demonstrations, adding further details, and correcting some inaccuracies: Lederman and Taylor [15] observed that perception of roughness was related to the spatial properties of a sample's surface and that tactile perception was not significantly affected by the relative velocity between finger and sample. The works of Lederman et al. [16] confirmed that, in the case of very coarse textures $(>630 \mu \mathrm{m})$, vibrational contribution does not play a significant role in tactile perception. In subsequent studies [6,7,17-19], it has been found that, if the sample texture is too small to deform the skin of the finger significantly, the spatial coding fails and texture must be perceived in a nonspatial way. The experimental work of Hollins and Risner [13] highlighted the role of relative motion in tactile perception. When there is no relative motion, finer textures cannot be correctly perceived; coarse ones, however, can still be adequately characterized, although they are perceived as being less rough.

These results are consistent with the duplex theory. Indeed, in a static contact, only slow adapting units are excited and, while the perception of coarse textures is still possible thanks to the spatial deformation gradient, textures that are too fine cannot be fully identified. In contrast, when relative motion occurs, fast adapting units are activated by the friction induced vibrations in the finger skin, meaning that textures with a spatial period of less than $100 \mu \mathrm{m}$ can be discriminated [20].

Recent studies [20-22] also investigated the role of the fingerprint in the perception phenomenon, highlighting its filtering effect. Moreover, an analysis of the vibration spectra highlighted the fact that scanning the finger over a sample with a well-defined spatial period produces vibrations with a spectrum that is characterized by well-defined peaks in frequency. Depending on the ratio between the sample wavelength and the fingerprint one, different behaviours can been identified. In particular, when the sample wavelength is much smaller than that of the fingerprint, the spectrum depends mainly on the texture of the sample; however, when the sample wavelength is coarser than that of the fingerprint, the acceleration spectrum depends mainly on the fingerprint wavelength $[17,23]$. These results are in agreement with the

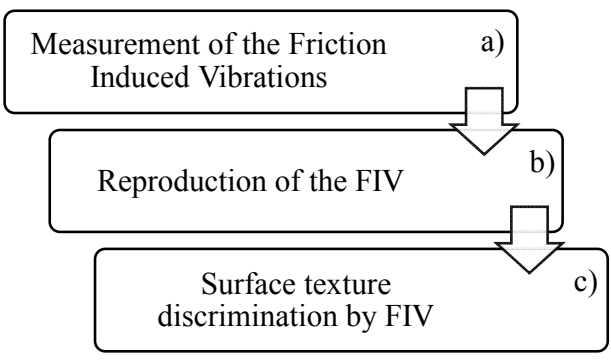

Fig. 1. Different steps in the analysis, measurement, and reproduction of vibration signals.

duplex theory and shine a light on the determinant role of the fingerprint spatial period, which behaves as a filter for induced vibrations.

In a more general way, an investigation of the tribological aspects and the spectra of induced vibrations could contribute to the definition of objective indexes related to the tactile perception of manufactured surfaces, including softness, hairiness [3], textile quality [3,24,25], texture, etc. Tactile perception has also, in large part, been investigated in order to evaluate the influence of other, different parameters including loads [26,27], pressure [28], finger inclination [29,30], finger orientation respect to rubbing direction [31], etc.

The purpose of this work is mainly to investigate the influence of the vibrations induced during finger/surface contact on the origin of tactile discrimination between different textures.

The paper is organized as follows. In Section 2, the methods employed to obtain the different measurements, and the different setups used for this purpose, are described. In Section 3.1, the tribological and dynamic signals that were measured are reported and analysed. In Section 3.2, the procedure to reproduce vibration signals that mimic the FIV is described and applied. In Section 3.3, the data collected on the tactile perceptions of a panel of subjects in response to the excitation signals generated in Section 3.2 is reported and discussed.

\section{Methods and materials}

The following steps were performed in order to evaluate the role that the vibrations induced by scanning a surface with the finger played in discriminating between different surface textures (Fig. 1):

- The vibrations induced by touching the sample surfaces were measured under controlled boundary conditions, using a panel of subjects.

- Equivalent excitation signals were developed to obtain the same vibrations that had been measured while they were touching the surfaces; for the same purpose, the $F R F$ (frequency response function) at the fingertip was also calculated.

- The equivalent excitation signals were applied to the subjects' fingertips in order to evaluate their ability to distinguish between the samples based solely on the reproduced vibration stimuli. 


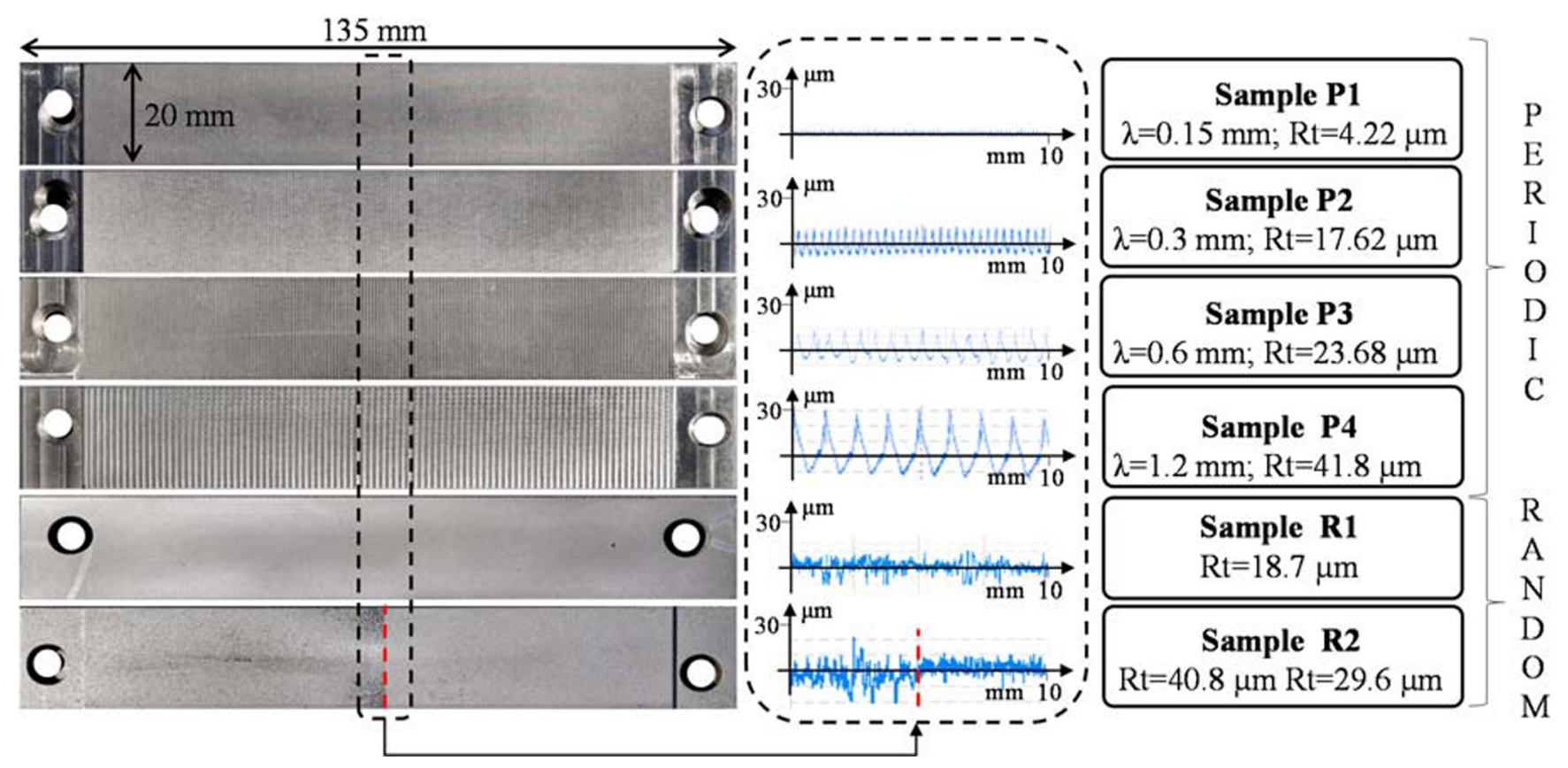

Fig. 2. Samples with different roughnesses and related profilometer traces.

The work presented in this paper was structured in different phases, each of which was characterized by a welldefined procedure and required a dedicated arrangement of the test equipment. All of the procedures and their respective setups are described in this section.

\subsection{Measurement of the FIV: methodology and setup}

The first step involved measuring the signals produced when the finger slid over a sample with a well-defined roughness. To this end, six samples (steel bars measuring $135 \times 20 \times 8$ $\mathrm{mm}$ ) with different roughnesses were used. Four of the samples (P1, P2, P3, P4) had a regular periodic roughness with a well-defined spatial period $\lambda$ and total height of the roughness profile $R_{t}$, that is, the vertical distance between the maximum profile peak height and the maximum profile valley depth along the length of the test zone (Fig. 2). The other two samples (R1, R2) were characterized by randomly distributed roughnesses (Fig. 2). The R2 sample was composed of two zones, each with a different roughness (Fig. 2). The periodic roughnesses were obtained by means of a milling process using different widths of the milling tool. The random roughness, on the other hand, was accomplished by means of a sandblasting process with different bead sizes and working pressures.

Figure 3 shows the TriboTouch setup, the experimental device used to perform the measurements. In particular, Figure $3 \mathrm{a}$, in addition to the chain measurement, shows the TriboTouch equipped for measurements from a Fake finger, which could also be of interest in terms of measurement automatisation. Conversely, Figure 3b shows the arrangement for measurements from a Real finger, as in the case of this paper. The sample was placed on a support frame, which was translated horizontally by a compliant mechanism (Fig. 3). The compliant mechanism was composed of two double parallelogram linkages, tilted towards each other (Fig. 3, on the right). Instead of bearings, the rigid sides of the parallelograms were linked by means of compliant joints, which exhibit no friction losses or noise [32]. The displacement of the sample was achieved by means of a linear voice coil actuator, where the relative motion between the coil and the magnet was obtained without direct contact. The actuator was driven by a controller connected to a computer, which made it possible to control the velocity and keep it constant during sliding. The choices of both the compliant mechanisms and voice coil actuator met the requirement of avoiding parasitic vibrations coming from the experimental setup, which could have significantly affected the measurements, due to the very small amplitudes of the induced vibrations on the finger. A TTL linear encoder (Fig. 3), whose resolution was $0.5 \mu \mathrm{m}$, provided the position feedback.

The main purpose of the measurement was to acquire information on the dynamics at the contact region between finger and sample. Unfortunately, it was impossible to get this information directly, without modifying the contact conditions themselves. For this purpose, a 1-axis accelerometer was fixed at the closest zone to the contact region, the fingernail (Fig. 4). The accelerometer recorded the acceleration along the direction perpendicular to the fingernail. Even if there is a filtering effect caused by the finger itself, knowing the $F R F$ of the finger makes it possible to recover, to a great extent, the actual dynamics at the contact region, as explained in the next section.

The insertion error caused by the presence of the accelerometer was negligible, thanks to its reduced mass. This was confirmed by comparative measurements taken using a laser vibrometer [33].

During the measurement, the fingertip was kept stationary while the sample surface was moved, thereby achieving what is known as the condition of "passive touch" (as opposed to "active touch", where the fingertip is 


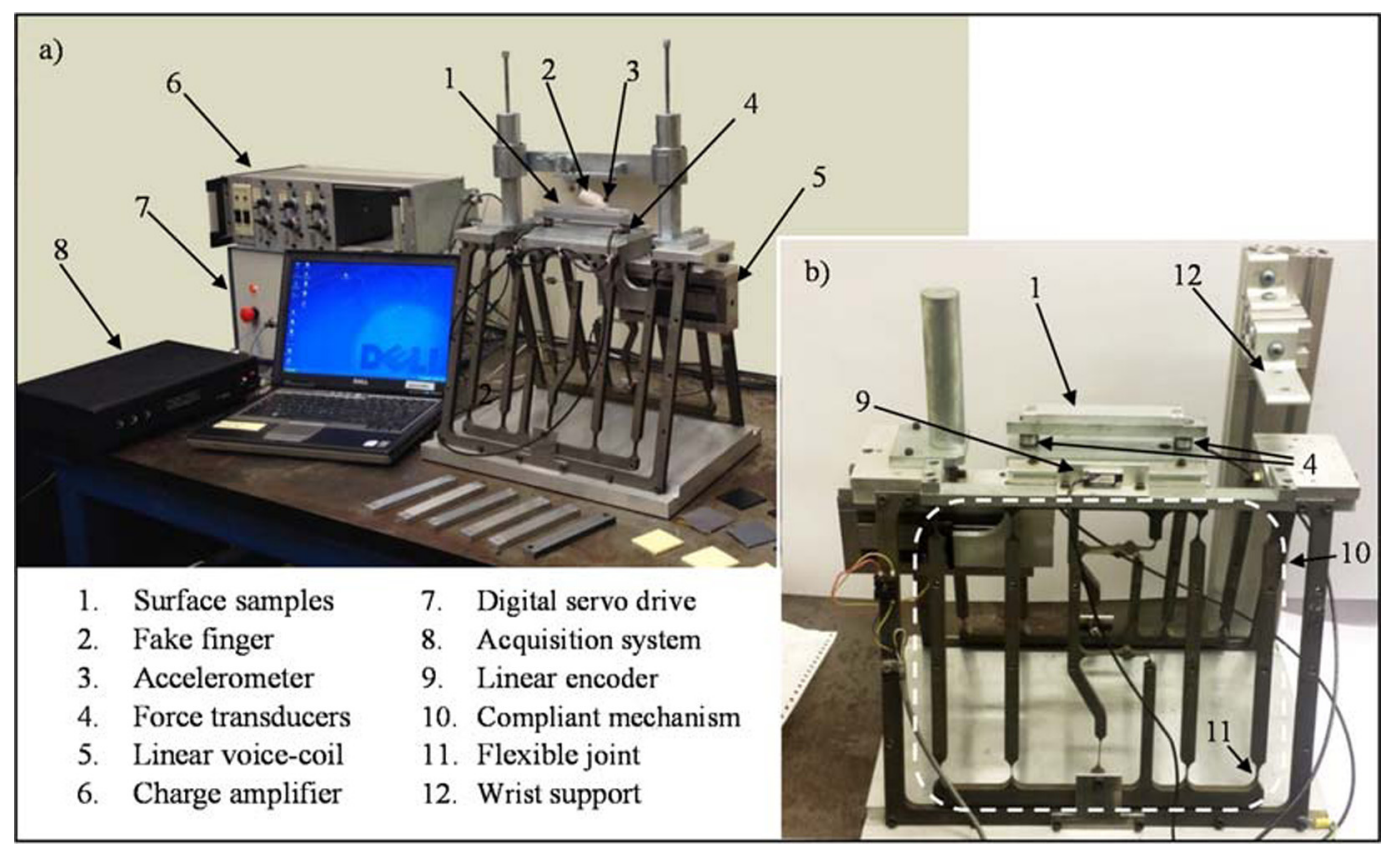

Fig. 3. TriboTouch experimental setup: (a) chain measurement with TriboTouch equipped for measurements from Fake finger and (b) TriboTouch arranged for Real finger measurement.

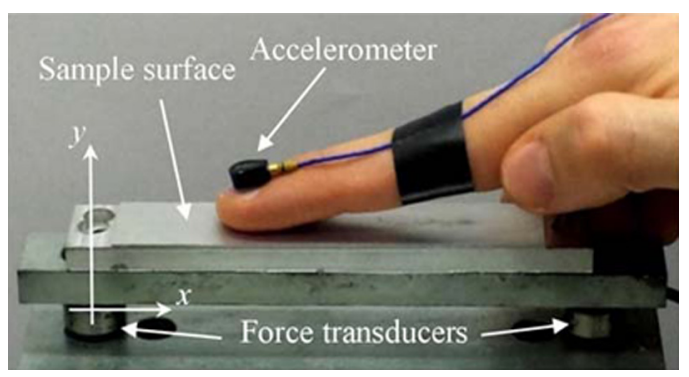

Fig. 4. TriboTouch, finger-sample test configuration.

moved). In this manner, the operator had better control over the normal force, which was maintained approximately constant at $0.5 \mathrm{~N}$ during the translation. Moreover, the translation velocity $v$ could be maintained at a constant value of $20 \mathrm{~mm} / \mathrm{s}$ by the voice-coil actuator. The values of $0.5 \mathrm{~N}$ and $20 \mathrm{~mm} / \mathrm{s}$ were chosen in order to reproduce the mean force and velocity values applied when scanning a surface with the fingertips [20]. The forces exchanged by the finger and the surface were measured by two triaxial force transducers (Fig. 4). Normal (along $y$ ) and tangential (along $x$ ) forces make it possible to evaluate the mean friction coefficient during the sliding contact. The transducers were connected to charge amplifiers and thence to the acquisition system.

The wrist of the operator rested on a rigid support in order to prevent undesired movements and to better control the normal force (Fig. 3). The finger was maintained at an angle of approximately $20^{\circ}$ with respect to the horizontal plane. Moreover, to avoid alterations in the contact conditions, the fingertip was cleaned with hand-cleansing gel and the sample surface was cleaned with alcohol before each test.

\subsection{Reproduction of the FIV signals: methodology and setup}

During frictional contact with a surface, the fingertip is stimulated by both normal and tangential contact stresses. These distributed exciting forces change both in time and in space depending on skin morphology and kinematics, which determine the real contact area and the related contact pressures. This makes it difficult to record and reproduce the distributed actual contact interaction.

Nevertheless, the skin acts as a membrane by transmitting the vibrations, excited at the contact interface, to the fingernail, where the accelerometer is placed. Even if the local spatial distribution of the contact is lost, the measurements of the acceleration at the fingernail make it possible to recover the amplitude and frequency distribution (spectrum) of the overall contact induced vibrations.

The goal of this work is to evaluate to what degree a difference in the spectrum of the vibrations induced by scanning an object with the fingertip can be perceived as a distinctive signal and allow for discrimination between different surfaces.

This section describes how the signals measured on the fingernail are used to generate an equivalent vibration signal which, sent to the fingertip by means of a dynamic exciter, makes it possible to obtain the same accelerations (frequency content, root mean square) measured at the fingernail.

\subsubsection{Evaluation of the finger FRF}

The first step is to determine the FRF of the finger. The finger can actually be viewed as a dynamic system, where the input is the force $(F)$ coming from the frictional contact 


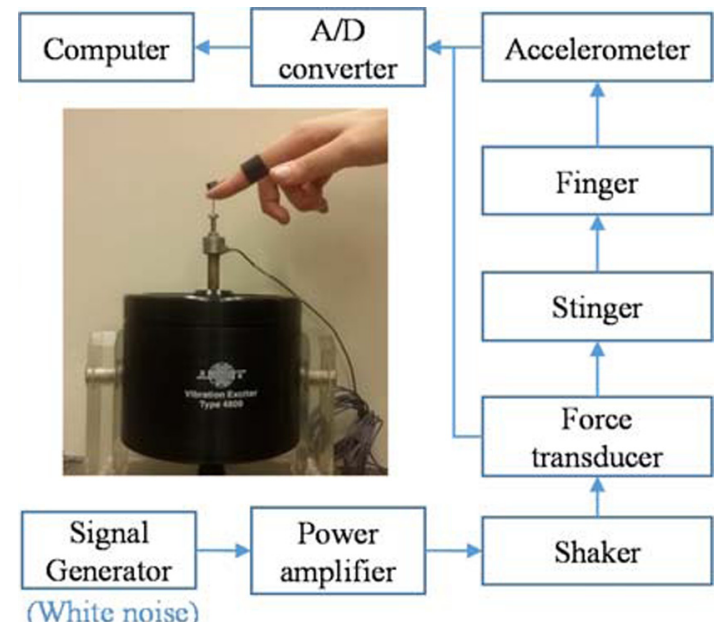

Fig. 5. FRF measurement setup.

and the output is the acceleration $(\ddot{X})$ measured on the nail (Fig. 4); so, strictly speaking, the FRF is an accelerance $A$ :

$$
A(\omega)=\frac{\ddot{X}(\omega)}{F(\omega)}
$$

Given that the system is linear, a first approximation of the transfer function of the finger can easily be obtained by measuring the force and acceleration. Figure 5 shows the setup used for this purpose.

Specifically, a signal generator sends a signal, opportunely amplified, to a shaker. The force is measured by means of a force transducer screwed in at the top of the shaker. A stinger is set up on top of the transducer. The fingertip rests on the stinger, maintaining a routine angle of $20^{\circ}$, and the acceleration is measured at the fingernail. This procedure was repeated for all the participants and the accelerance was determined for each of them.

In this configuration, the measured force is a normal force. The accelerance provides the relationship between a normal force exciting the finger and the acceleration measured in the direction perpendicular to the nail. Obviously, in real contact, the tangential components of the force and their spatial distributions also play a role [34]. However, in light of the fact that the aim of the present analysis is to estimate the influence of the frequency content of the FIV on tactile recognition of textures, and the influence of the frequency content of the stimulus on tactile perception, this shortcoming does not represent a lack of accuracy. On the contrary, in this way, only the contribution of the frequency content is taken into account, without the spatial distribution and the overall force direction being reproduced. Thus, the reconstructed signal will have the same frequency content as the actual force exchanged between the finger and the sample. We plan to take the effect of the force direction with respect to the finger and the pressure distribution into consideration when further developing this work.

Using the procedure described above, the FRFs of each subject were obtained; an example is presented in Figure 6. The same figure also reports coherence, which measures the correlation between the input signal $(F)$ and the output signal $(\ddot{X})$, making it possible to evaluate whether external noise has affected the measurement.

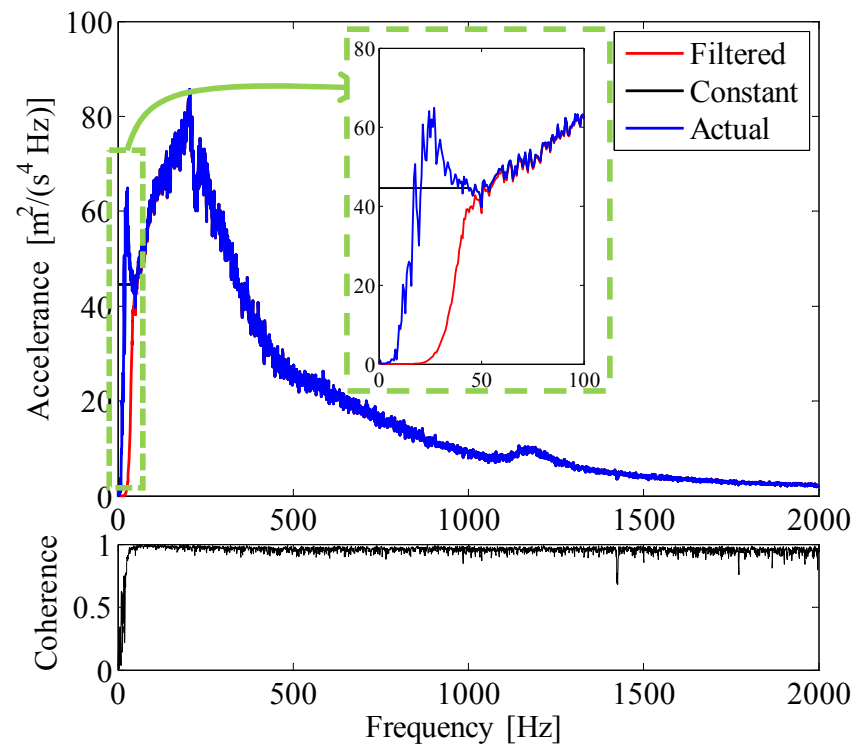

Fig. 6. Finger frequency response function.

The FRF shows its greatest increase at around $200 \mathrm{~Hz}$, which is followed by a subsequent decrease moving towards the higher frequencies. We can also observe that the coherence remains close to 1 for a large range of frequencies, except for values greater than $2000 \mathrm{~Hz}$ and lower than $40 \mathrm{~Hz}$. At high frequencies, the loss of coherence is mainly ascribable to nonlinearities of the system and to large damping; nevertheless, the range of interest for the tactile issue remains under about $500 \mathrm{~Hz}$.

The drastic drop in coherence at low frequencies was related to the intrinsic limits of the excitation system. Indeed, the shaker presents a cut-off frequency of around $30 \mathrm{~Hz}$, with a response curve that reaches a stable response at about $40 \mathrm{~Hz}$. This issue led to the reduction of the frequency range of the analysis at over $40 \mathrm{~Hz}$. The immediate consequence of this choice was the need to filter the FRF (Fig. 6) in the range between 0 and $40 \mathrm{~Hz}$, in order to avoid the reproduction of fake signal contents below $40 \mathrm{~Hz}$. Two possible solutions were considered: (a) high-pass filtering with frequency cut-off at $40 \mathrm{~Hz}$ (red line in the inset of Fig. 6) and (b) constant value of the FRF between 0 and $40 \mathrm{~Hz}$ (black line in the inset of Fig. 6).

Solution (a), although it seems to be the simplest, could not be used. As a matter of fact, because the final signal is obtained by means of the inversion of the relationship (1), as explained later, a small value of $A(\omega)$ gives rise to overestimated values of $F(\omega)$, entailing the accentuation of low frequency effects. Consequently, solution (b) was used in the following analysis. A direct validation of the methodology has been performed, comparing the acceleration signals obtained using both methods with the original signal data.

Obviously, this limits the validity of the conclusions of this work to a reduced frequency range $(40-2000 \mathrm{~Hz})$. Nevertheless, the response of the FA II and SA II units, which are responsible for perceiving medium-high frequency transients, falls within this range.

The use of a stinger instead of a flat surface fully satisfies the need for better coherence. Nevertheless, measurements with the fingertip placed directly on a flat surface were also 
carried out, so as to verify the influence of fingertip deformation on the FRF; the FRFs thus obtained showed no appreciable differences. A sampling frequency of $5000 \mathrm{~Hz}$ was used, in order to obtain the spectrum of the signal up to $2500 \mathrm{~Hz}$.

\subsubsection{Determination of the equivalent excitation signal}

The goal of this phase is to evaluate whether the vibration signal, when applied directly to the fingertip by a dynamic exciter, produces the same accelerations as the ones experienced during the frictional sliding of the fingertip on the touched surface. Although the TriboTouch provides the global tangential and normal force signals (Fig. 4), the vibrations measured on the finger were chosen as the reference signal to be reproduced. This choice is motivated by the fact that the acceleration measured on the finger has the vibrational content closest to the one perceived by the mechanoreceptors within the fingertip skin.

The equivalent excitation signal was obtained from the response signals and the calculated FRF. Indeed, the equivalent normal force excitation, $F_{\text {Neq }}$, could be calculated by using the measured signals of acceleration at the fingernail and the FRF of the finger.

The equivalent normal force $F_{N e q}$ makes it possible to reproduce a frequency content similar to the real contact force. Thus, starting with the signals of acceleration measured during sliding of the finger over the different surface samples, the equivalent normal force $F_{N e q}(\omega)$ was calculated in the frequency domain:

$$
F_{N e q}(\omega)=\frac{\ddot{X}(\omega)}{A(\omega)}
$$

Finally, the spectra of the equivalent normal forces were opportunely calculated and an inverse fast Fourier transform (IFFT [35]) was applied in order to obtain the signals in the time domain $F_{N e q}(t)$.

The variables in the relationship (2) were considered in terms of both their real and imaginary parts. In this manner, the procedure accounted for the phase of the signal and, once the IFFT was applied, the time evolution of the signal was reconstructed. Once the equivalent normal forces had been calculated in the time domain, they were used as an input to excite the fingertip (see Sect. 2.3). More precisely, they were normalized and, by means of the signal generator, they were sent to the shaker in order to be perceived by the subject's fingertip.

Preliminary tests were required to evaluate the suitable gain to apply at the power amplifier for each sample, in order to obtain the same value of acceleration RMS as had been measured in Section 2.1.

This operation was repeated for each sample and for each subject. At the end of this phase, all the $F_{N e q}$ signals and their related gains were available to be reproduced by the shaker and applied to the fingertips of the subjects.

\subsection{Surface discrimination by FIV: methodology and setup}

In order to obtain preliminary feedback to evaluate the actual influence of the FIV on the discrimination of object surfaces by tactile perception, an investigation campaign

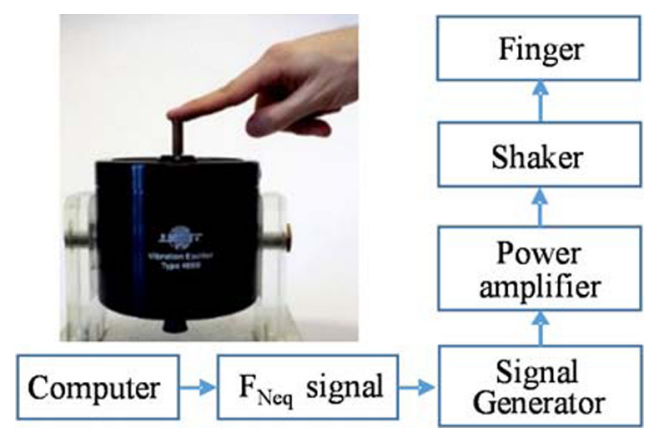

Fig. 7. Test B: surface discrimination through FIV.

was conceived and conducted on a panel of 10 subjects. All of the subjects were between 24 and 40 years of age, with an average age of 28 , and none of them reported diseases that could affect perception capabilities. The panel was composed of 7 men and 3 women, all volunteers and all right-handed. The essence of the test was to compare the sensation perceived while sliding the fingertip over the sample to that experienced when resting the fingertip on the shaker (fed by the $F_{N e q}$ ). The purpose was to examine whether or not subjects were able to match each signal to the corresponding sample from which it had been derived. As noted in Section 2.2.2, since the $F_{\text {Neq }}$ has the same frequency content of the actual signal, this investigation should help to understand how the spectrum of the FIV contributes to the tactile discrimination of objects' surfaces.

Following the calculation of the required signals (reported above) for all of the samples and all of the subjects, this part of the work was divided into three main phases:

- Phase no. 1: Each subject rubbed his finger over each sample, to acquire familiarity with the perception provided by the samples and to link a sample with the correspondent tactile sensation. Care was taken to train the subjects to reproduce, as closely as possible, the same scanning velocity experienced on the TriboTouch $(20 \mathrm{~mm} / \mathrm{s})$.

- Phase no. 2, test A: Each subject rubbed his finger over each sample, as before, but in this phase the samples were hidden from the subject's view and presented to the subject in a random order. The participant had to express his opinion regarding which of the samples he was touching. A table was filled out for each participant, reporting his right and wrong answers. This phase could be repeated in case of an excessive number of wrong connections, in order to improve the subject's familiarity with the samples.

- Phase no. 3, test B: Each subject laid his finger on a flat surface (of $\sim 1 \mathrm{~cm}^{2}$ ) on the top of the shaker (as in Fig. 7) which was fed by a series of $F_{N e q}$ signals in a random sequence. In this phase as well, a table was filled out for each participant, with the $F_{N e q}$, sent to the shaker (and unknown to the participant) and the sample stated by the participant. The participants were also permitted to evaluate each signal several times if doubts persisted. During the test, the volunteers wore headphones, to avoid being influenced by acoustic signatures from the 


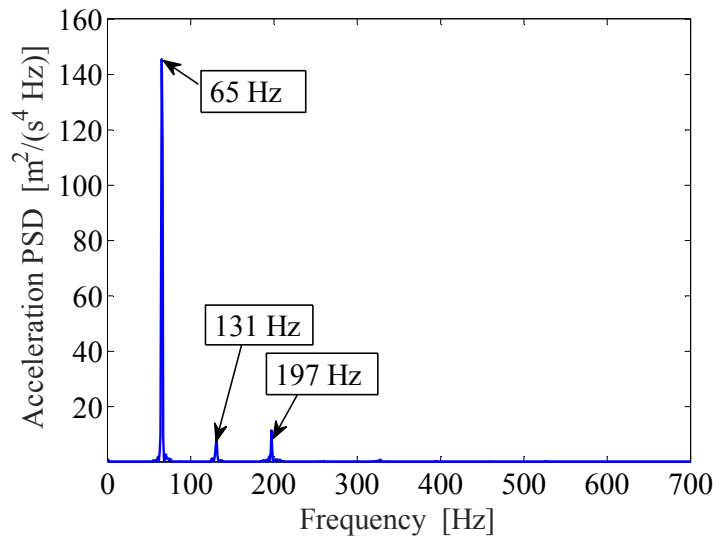

(a)

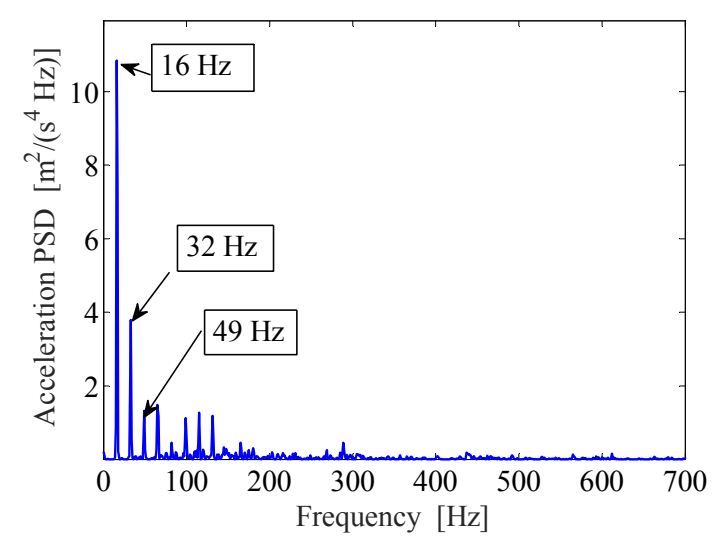

(b)

Fig. 8. Acceleration power spectral density (PSD): (a) sample P2 and (b) sample P4.

shaker. In addition, they were permitted to move their finger, and their arm, over the shaker, in order to take into account kinesthesis and simulate, as closely as possible, the original haptic act.

The results of this test were used to compare the ability of the volunteers to discriminate between the surface samples by means of the sensation perceived when touching the actual samples with their ability to do so through the sensation experienced when putting the finger over a shaker fed by the samples' respective $F_{N e q}$ signals.

\section{Results and discussion}

\subsection{Measurements of the FIV}

Using the TriboTouch setup, the force and acceleration signals were recorded when each of the six samples in Figure 2 slid under the fingertip, with controlled boundary conditions (normal force $0.5 \mathrm{~N}$, translation velocity $v=20 \mathrm{~mm} / \mathrm{s}$, a finger angle of about $20^{\circ}$.

Figure 8a presents the power spectral density (PSD) of the acceleration measured with sample P2.

This figure and the following ones show the PSD of the signals obtained for one subject, making it possible to focus on the main characteristics of the frequency content. The spectrum shows a peak at $65 \mathrm{~Hz}$ and superharmonics at 131 and $197 \mathrm{~Hz}$; the main harmonic is directly related to the spatial wavelength $\lambda$ of the roughness of the sample and to the translation velocity $v$. The frequency at which the protrusions of the fingerprints intersect each solid summit of the roughness is approximately $v / \lambda$ (in the case of sample $\mathrm{P} 2$ it is $20 / 0.3=67 \mathrm{~Hz}$ ), which is in line with results from previous works $[18,19]$. Similar behaviour was also reported for the other periodic samples, as shown in Figure 8b, where the frequency of the main harmonic is smaller, due to the larger value of the spatial wavelength of the sample surface $\mathrm{P} 4(\lambda=1.2 \mathrm{~mm})$. In contrast, in the case of random samples, the spectrum does not present a main harmonic, as can be observed from Figure 9a for the sample R1.

In this case, the signal is instead characterized by a broadband frequency content, with substantial contribution around the $200 \mathrm{~Hz}$ mark. The same occurs for the R2 sample (Fig. 9b), except for the fact that the greatest energy contribution is in the range between 100 and $300 \mathrm{~Hz}$. The frequency band where the vibrational energy is distributed depends on both the topography of the surface and the scanning velocity. The vibration signal was recorded for each sample and for each subject.

\subsection{Reproduction of the FIV}

Once the acceleration on the fingernail has been measured during the scanning of the surface, and once the FRFs at the fingertip have been obtained, the signal to send to the shaker can be calculated in such a way as to reproduce the same accelerations on the fingernail. As described in greater detail in Section 2.2.2, the acceleration signals measured while touching the surface (hereafter called FIV acceleration) were used as the starting point to obtain the corresponding equivalent normal force $F_{N e q}$ signals. These signals were, in turn, sent to the shaker, resulting in a new measure of acceleration on the fingernail (hereafter termed $F_{\text {Neq }}$ acceleration). Special care was taken to obtain the same acceleration RMS value by means of the gain set in the power amplifier. The main goal of this step was to reproduce an excitation with a similar spectrum distribution and overall amplitude to the excitation arising from the actual scanning of the sample surface.

Figure 10, which presents data related to sample P1, shows an example of the comparison between the FIV acceleration (obtained by scanning the finger on the surface) and the $F_{\text {Neq }}$ acceleration (obtained by reproducing the equivalent excitation with the shaker). We can observe that the frequency content of the two signals is largely consistent.

The same is true of samples P2 and P3, as can be observed, for instance, in Figure 11a. In the case of the random samples, R1 and R2, the two accelerations again show excellent coherence, in both the frequency spectrum (in particular for the medium-high frequencies) and the energy distribution, at least in the frequency range that affects tactile perception (Fig. 11b). It is worth noting that, in the case of the samples R2, taking into account the time evolution (as described in Section 2.2.2), the reconstructed 


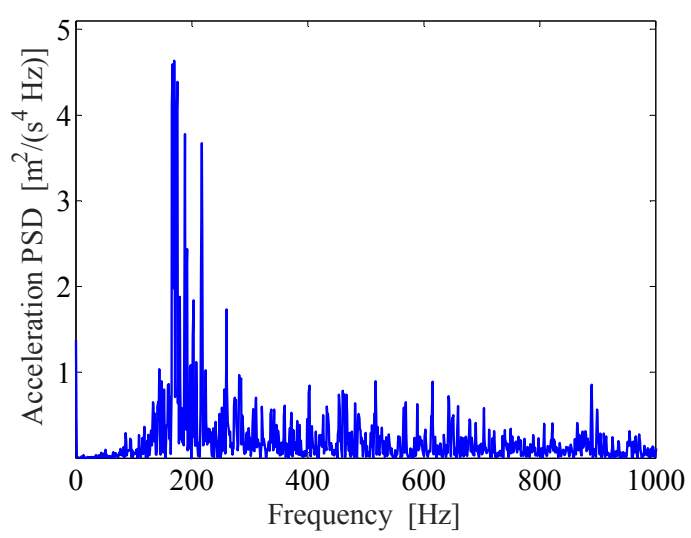

(a)

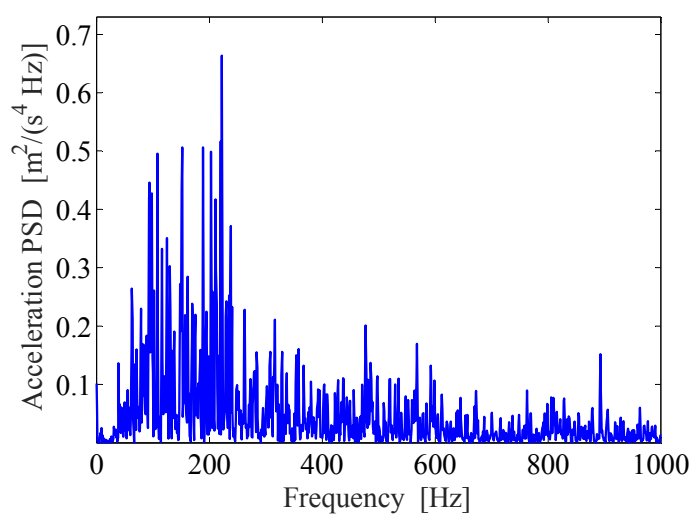

(b)

Fig. 9. Acceleration power spectral density (PSD): (a) sample R1 and (b) sample R2.

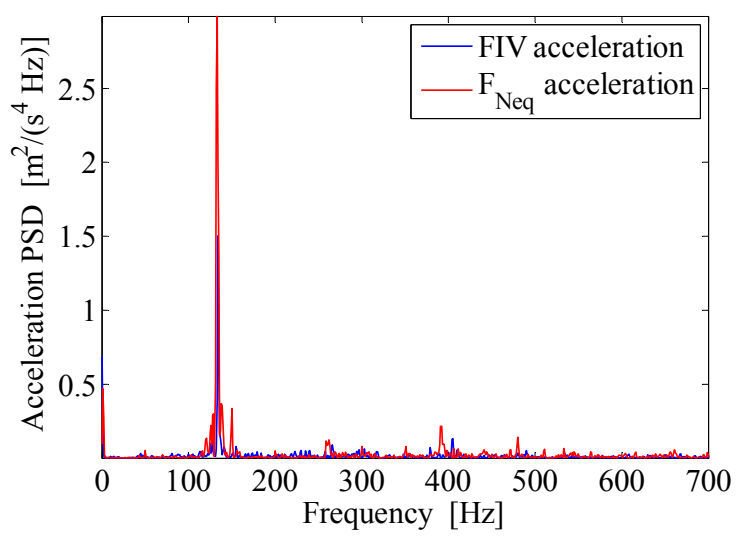

Fig. 10. Comparison of acceleration PSD for sample P1: FIV acceleration (blue line) and $F_{N e q}$ acceleration (red line).

signal is able to reproduce the change in the vibration signal experienced when passing over the two zones with different roughnesses. However, in the case of sample $\mathrm{P} 4$, the reproduced signal does not correspond to the original signal (Fig. 12).

In fact, as Figure 12 shows, the $F_{N e q}$ acceleration completely lacks low frequency harmonics. This is connected to the fact that the $\mathrm{P} 4$ sample has frequencies of interest that cover values lower than the shaker limit $(40 \mathrm{~Hz})$. It has therefore been excluded from the following considerations and, in particular, from the subsequent investigation campaign.

The above results suggest that the use of the $F_{N e q}$ signal gives a sufficiently accurate reproduction of the overall vibrational response measured on the fingernail. This is primarily due to the use of the finger FRF, which allowed us to give the appropriate weight to the frequency content of the spectrum, which is generally filtered by the finger. Although the directionality and space distribution of the excitation at the contact cannot be reproduced, the frequency content of the vibrations induced on the fingertip is reproduced, in order to investigate its role in the ability to discriminate surface textures through friction induced vibrations.

\subsection{Surface texture discrimination by FIV}

As previously discussed, the last step of this work was an investigation campaign to examine the role FIV plays in tactile perception, and specifically on the ability to recognise different surface textures using only overall FIV input. Due to the restricted number of participants, this campaign does not have sufficient statistical robustness to infer strong conclusions. Its role has been primarily to provide recommendations for conceiving and defining the protocol of the campaign and to shine a light on the drawbacks of the procedure. While the results obtained are useful for a direct comparison between the tested surfaces, their limits are noted at the end of this section.

The volunteers performed the tests on five samples, P1, P2, P3, R1, and R2 (Fig. 2), in compliance with the procedure described in Section 2.3. Sample P4 was excluded due to the impossibility of reproducing the FIV signal with the shaker used. The results of this test have been treated statistically and summarized using the association matrix reported in Figure 13. The matrix was built in the following manner: the $F_{N e q}$ signals, corresponding to each surface sample and used to feed the shaker, are in the abscissa; in the ordinate, the sample indicated by the volunteer as being the one that provided the same sensation perceived during the active touch is reported. The squares along the matrix diagonal (green dotted line) indicate that the sample was correctly recognised by the volunteer based solely on the FIV signal. A square out of the diagonal indicates misidentification of a sample. The colour of each square is directly proportional to the association degree, which indicates the percentage of participants that gave that answer, according to the scale on the left.

Figure 13 shows periodic sample P2 as having the maximum association degree (50\%); in other words, $50 \%$ of the participants correctly associated the $F_{N e q}$ signal of P2 with the P2 surface sample. The second-highest association degree, remaining on the diagonal that represents the locus of correct associations, was for periodic sample P1 and random sample $\mathrm{R} 2$, both of which showed an association degree of $40 \%$. They were followed by periodic sample P3 $(30 \%)$ and random sample R1 (20\%). 


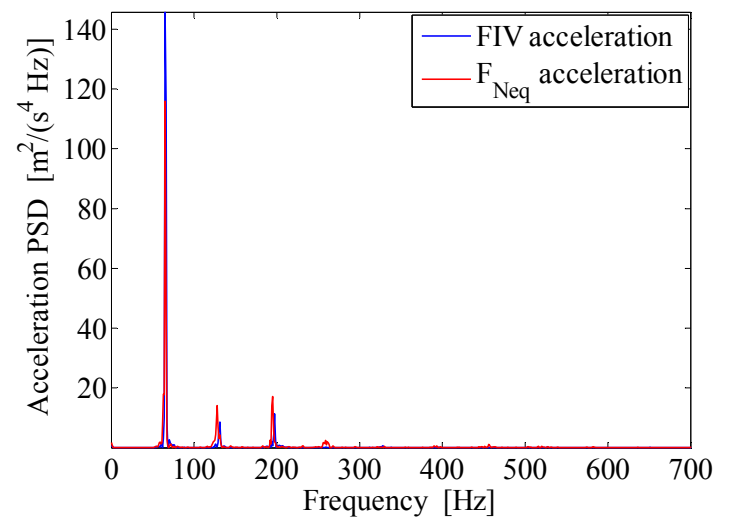

(a)

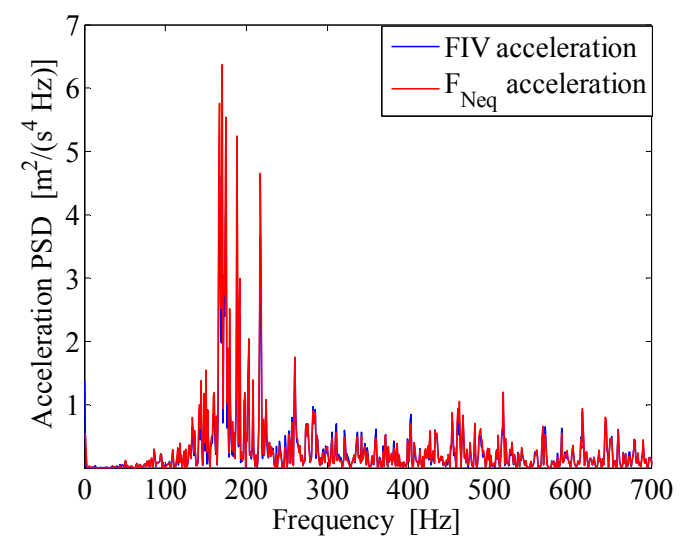

(b)

Fig. 11. Comparison between acceleration PSD: (a) sample P2 and (b) sample R1.

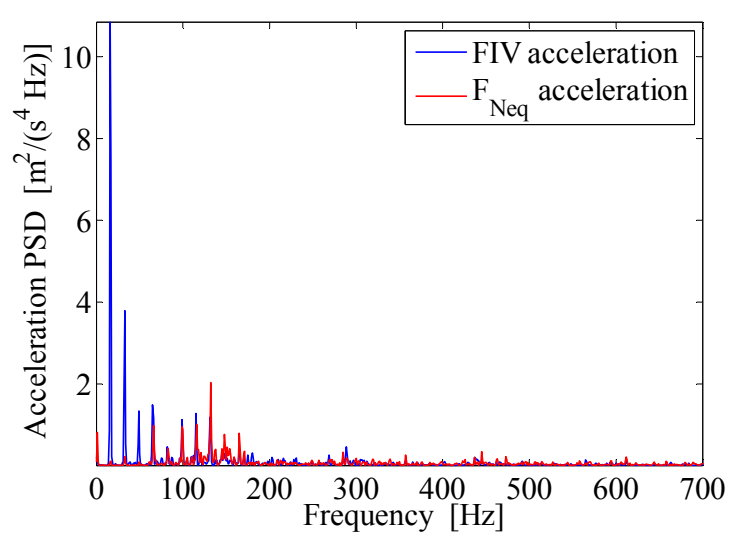

Fig. 12. Acceleration PSD of sample P4.

In agreement with Katz's theory [12], the greater degrees of association were observed for the periodic samples, and in particular for the ones with a finer texture. Indeed, samples P1 and P2 have roughness wavelengths (Fig. 2) closer to values that, in the double perception theory, are considered as representative of "fine textures". In contrast, sample P3 $(\lambda=0.6 \mathrm{~mm})$ can be considered to be in the range of "coarse textures" [20,22] and a lower value of correct association was obtained.

In these tests, the only information provided by the $F_{N e q}$ signal was related to the vibrations induced during the actual sliding of the fingertip on the sample surface. No information about the static deformation of the finger skin was reproduced. In the case of "fine textures", the vibrational information is meaningful for recognising the surface samples. This confirms that, in the perception of fine structures, a prominent role is played by the vibrational component rather than the static deformation of the skin. In contrast, for a coarse texture (P3), the vibrational signal is too poor in information for surface recognition to be possible.

If we compare the random samples, the highest association degree was recovered for sample R2. This can be attributed to the enhanced identification ability resulting from the presence of a discontinuity within the perceived vibrational stimuli, caused by the sequence of two distinct

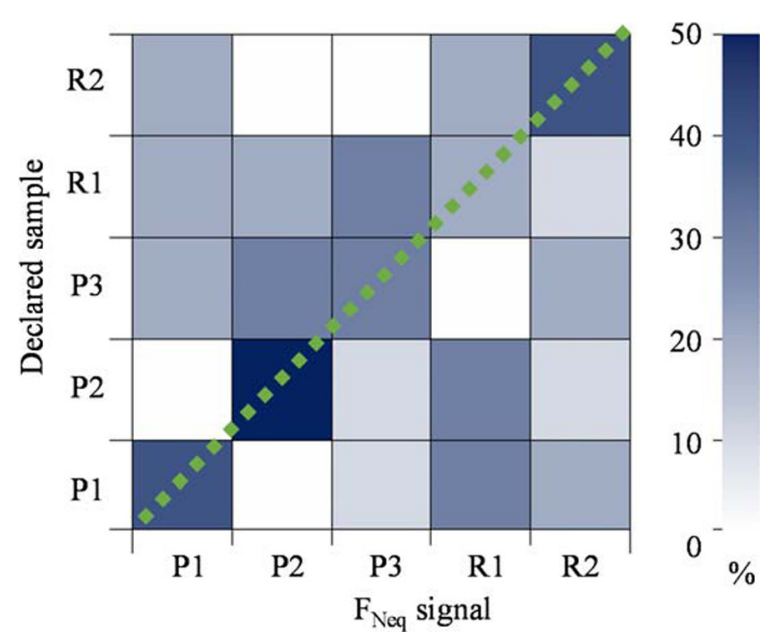

Fig. 13. Association matrix. "-Line of correct association.

surface roughnesses on the same sample (Fig. 2). The volunteers were able to "feel" the passage between the two halves of the surface, characterised by different roughnesses, thanks to the variation in the reproduced $F_{N e q}$ signal.

The other random sample, R1, exhibits the lowest value of association degree. This is probably due to the fact that a lack of the sort of regular frequency pattern found on the periodic samples makes the signal less identifiable and its perception more susceptible to error. In addition, we observed that the subjects often associated the R1 sample with periodic samples with finer textures. This is probably because the frequency content of the random $\mathrm{R} 1$ samples is quite concentrated, in a frequency band of between approximately 100 and $300 \mathrm{~Hz}$, where the main frequency peaks of the finer periodic samples are also located.

Foremost among all our observations so far is the fact that even in the best case, as Figure 13 reveals, the maximum association degree value remains low (50\%). This can be attributed to different sources of bias, which could have some misleading effect on the ability of the subjects to discriminate between the reproduced tactile signals. One of these sources of error could be the 
unavoidable difference between the rubbing velocities in the passive touch measurements on the TriboTouch and the active touch of the samples in phase 3 ; this despite the fact that, as described in Section 2.3, special care was taken to train the subjects to reproduce the same velocity. Other bias factors could arise from the force direction, restricted to the normal one, and from the movement of the finger over the shaker, which, while it did account for kinesthesis, could also have introduced some additional stimulus. Moreover, kinesthesis could play a fundamental role, one which was not fully taken into account in this study, given that it lacked the correlation between the kinematics of the limb and the transient variability of the spectrum. This is one of the reasons why the measurements were made at a constant speed. In addition, of course, the variability of the kinematics could be one of the biases of the low discrimination performances. Accounting for the correlation between limb kinematics and spectrum is an extreme challenging issue and will certainly be the object of future study. Finally, there are factors other than surface roughness that contribute to tactile perception [36,37].

\section{Conclusions}

This paper presents an experimental analysis of the dynamic aspects of the phenomena that occur when a finger slides over a surface. The main purpose of this work is to investigate the role of the friction induced vibrations that are generated when touching a surface on the perception of surface textures. For this purpose, after having taken measurements of FIV on specific surface samples, this work was devoted to conceiving of, defining and applying a method for reconstructing the signals associated with the forces experienced upon contact between the finger and the samples. These are forces that are inaccessible for direct measurement. At this stage, our analysis has been focused on the reproduction of the frequency content of the force signals, regardless of force direction and spatial distribution.

A preliminary campaign was conducted, involving a panel of volunteers, to compare the perception experienced when resting the finger on a shaker fed with the reconstructed force signals with the perception experienced when sliding the finger over the surface samples. This experiment allowed us to focus our attention on the effects of the frequency distribution of friction induce vibrations, ignoring the quasi-static deformation of the skin and other perception mechanisms that come into play in the case of direct contact with surface samples. While the restricted number of subjects does limit the statistical robustness of the results, the tests performed are useful for making direct comparisons between the tested surfaces and how they are perceived.

The results showed that the maximum values for the ability to associate the equivalent signal with the real one occur in correspondence to samples with finer periodic textures. Thus, in the case of "fine textures", information containing solely the dynamic aspect of the tactile experience is meaningful for discriminating between the textures touched. On the other hand, when the textures become coarser, the lack of other information, such as the spatial deformation of the skin, limits the correct identification of samples. This is consistent with Katz's duplex perception theory. In the case of a surface with a non-periodic roughness, the absence of a recognisable frequency pattern seems to make the perception more sensitive to errors. Moreover, the identification of a surface sample can be improved by discontinuities of the surface texture within the same sample surface, which is "felt" by the participants during the application of the equivalent dynamic stimuli.

Although the results obtained through this analysis suggest that the role played by the spectral distribution of friction induced vibrations is a fundamentally important one, they also reveal that, given the complexity of the phenomenon, the reproduction of vibrational signals alone cannot permit satisfactory recognition of the entire panel of tested textures. Further investigation is needed, both to examine the effects of other factors, such as vibration amplitudes, force direction, and the kinematics of the fingertip, as well as to analyse the effects of differences in the spectrum distribution on a larger panel of samples including, for instance, textiles and soft materials.

\section{References}

[1] E. Lumpkin, M. Caterina, Mechanisms of sensory transduction in the skin, Nature 445 (2007) 858-865

[2] S. Choi, K.J. Kuchenbecker, Vibrotactile display: perception, technology and applications, Proc. IEEE 101 (2013) 2093-2104

[3] M.A. Bueno, B. Lemaire-Semail, M. Amberg, F. Giraud, Pile surface tactile simulation: role of the slider shape, texture close to fingerprints, and the joint stiffness, Tribol. Lett. 59 (2015) 1-12

[4] S. Asano, S. Okamoto, Y. Matsuura, H. Nagano, Y. Yamada, Vibrotactile display approach that modifies roughness sensations of real textures, in: Proceedings of 2012 ROMAN, IEEE, Paris, France, 2012, pp. 1001-1006

[5] M. Biet, F. Giraud, B. Lemaire-Semail, Implementation of tactile feedback by modifying the perceived friction, Eur. Phys. J. Appl. Phys. 43 (2008) 123-135

[6] K.O. Johnson, The roles and functions of cutaneous mechanoreceptors, Curr. Opin. Neurobiol. 11 (2001) 455-461

[7] T. Maeno, K. Kobayashi, N. Yamazaki, Relationship between the structure of human finger tissue and the location of tactile receptors, JSME Int. J. C: Mech. Syst. Mach. Elem. Manuf. 41 (1998) 94-100

[8] S.J. Bolanowski, G.A. Gescheider, R.T. Verrillo, C.M. Checkosky, Four channels mediate the mechanical aspects of touch, J. Acoust. Soc. Am. 84 (1988) 1680-1694

[9] K.O. Johnson, T. Yoshioka, F. Vega-Bermudez, Tactile functions of mechanoreceptive afferents innervating the hand, J. Clin. Neurophysiol. 17 (2000) 539-558

[10] A. Brisben, S. Hsiao, K. Johnson, Detection of vibration transmitted through an object grasped in the hand, J. Neurophysiol. 81 (1999) 1548-1558

[11] S.J. Bolanowski, G.A. Gescheider, R.T. Verrillo, Hairy skin: psychophysical channels and their physiological substrates, Somatosens. Motor Res. 11 (1994) 279-290

[12] D. Katz, The world of touch, Psychology Press, New York, USA, 2013 
[13] M. Hollins, S.R. Risner, Evidence for the duplex theory of tactile texture perception, Percept. Psychophys. 62 (2000) 695-705

[14] M. Wiertlewski, J. Lozada, V. Hayward, The spatial spectrum of tangential skin displacement can encode tactual texture, IEEE Trans. Robot. 27 (2011) 461-472

[15] S.J. Lederman, M.M. Taylor, Fingertip force, surface geometry, and the perception of roughness by active touch, Percept. Psychophys. 12 (1972) 401-408

[16] S.J. Lederman, J.M. Loomis, D.A. Williams, The role of vibration in the tactual perception of roughness, Percept. Psychophys. 32 (1982) 109-116

[17] M.A. Srinivasan, R.H. LaMotte, Encoding of shape in the responses of cutaneous mechanoreceptors, in: O. Franzen, J. Westman (eds.), Information processing in the somatosensory system, Macmillan Education UK, London, 1991, pp. 59-69

[18] H. Culbertson, J. Unwin, B.E. Goodman, K.J. Kuchenbecker, Generating haptic texture models from unconstrained tool-surface interactions, in: Proceedings of World Haptics Conference (WHC), IEEE, Daejeon, South Korea, 2013, pp. 295-300

[19] H. Culbertson, J. Unwin, K.J. Kuchenbecker, Modeling and rendering realistic textures from unconstrained tool-surface interactions, IEEE Trans. Haptics 7 (2014) 381-393

[20] R. Fagiani, F. Massi, E. Chatelet, Y. Berthier, A. Akay, Tactile perception by friction induced vibrations, Tribol. Int. 44 (2011) 1100-1110

[21] R. Fagiani, F. Massi, E. Chatelet, J. Costes, Y. Berthier, Contact of a finger on rigid surfaces and textiles: friction coefficient and induced vibrations, Tribol. Lett. 48 (2012) $145-158$

[22] J. Scheibert, S. Leurent, A. Prevost, G. Debregeas, The role of fingerprints in the coding of tactile information probed with a biomimetic sensor, Science 323 (2009) 1503-1506

[23] R. Klatzky, S. Lederman, Tactile roughness perception with a rigid link interposed between skin and surface, Percept. Psychophys. 61 (1999) 591-607 (in English)

[24] I. Koc, C. Aksu, Tactile sensing of constructional differences in fabrics with a polymeric finger tip, Tribol. Int. 59 (2013) 339-349

[25] M.A. Bueno, B. Lamy, M. Renner, P. ViallierRaynard, Tribological investigation of textile fabrics, Wear 195 (1996) $192-200$
[26] W. Tang, S. Gel, H. Zhu, X. Cao, N. Li, The influence of normal load and sliding speed on frictional properties of skin, J. Bionic Eng. 5 (2008) 33-38

[27] J. Asserin, H. Zahouani, P. Humbert, V. Couturaud, D. Mougin, Measurement of the friction coefficient of the human skin in vivo: quantification of the cutaneous smoothness, Colloids Surf. B: Biointerfaces 19 (2000) 1-12

[28] S. Derler, U. Schrade, L. Gerhardt, Tribology of human skin and mechanical skin equivalents in contact with textiles, Wear 263 (2007) 1112-1116

[29] R.K. Sivamani, J. Goodman, N.V. Gitis, H.I. Maibach, Friction coefficient of skin in real-time, Skin Res. Technol. 9 (2003) 235-239

[30] J. van Kuilenburg, M. Masen, E. van der Heide, A review of fingerpad contact mechanics and friction and how this affects tactile perception, Proc. Inst. Mech. Eng. J.: J. Eng. Tribol. 229 (2015) 243-258

[31] A. Prevost, J. Scheibert, G. Debrégeas, Effect of fingerprints orientation on skin vibrations during tactile exploration of textured surfaces, Commun. Integr. Biol. 2 (2009) 422-424

[32] N. Lobontiu, Compliant mechanisms: design of flexure hinges, CRC Press, Boca Raton, FL, USA, 2002

[33] R. Fagiani, F. Massi, E. Chatelet, Y. Berthier, A. Sestieri, Experimental analysis of friction-induced vibrations at the finger contact surface, Proc. Inst. Mech. Eng. J.: J. Eng. Tribol. 224 (2010) 1027-1035

[34] A. Smith, C. Chapman, M. Deslandes, J. Langlais, M. Thibodeau, Role of friction and tangential force variation in the subjective scaling of tactile roughness, Exp. Brain Res. 144 (2002) 211-223

[35] J.W. Cooley, J.W. Tukey, An algorithm for the machine calculation of complex Fourier series, Math. Comput. 19 (1965) 297-301

[36] J. Dacleu Ndengue, I. Cesini, J. Faucheu, E. Chatelet, H. Zahouani, D. Delafosse, F. Massi, Tactile perception and friction induced vibrations: discrimination of similarly patterned wood-like surfaces, IEEE Trans. Haptics, 2017, accepted for publication, doi:10.1109/TOH.2016.2643662

[37] B. Delhaye, V. Hayward, P. Lefevre, J. Thonnard, Textureinduced vibrations in the forearm during tactile exploration, Front. Behav. Neurosci. 6 (2012) 60-69

Cite this article as: M. Di Bartolomeo, F. Morelli, D. Tonazzi, F. Massi, Y. Berthier, Investigation of the role of contact-induced vibrations in tactile discrimination of textures, Mechanics \& Industry 18, 404 (2017) 